\title{
Self-potential anomalies in some Italian volcanic areas
}

\author{
Rosa Di Maio, Vincenzo Di Sevo, Salvatore Giammetti, Domenico Patella, Sabatino Piscitelli \\ and Carmine Silenziario \\ Dipartimento di Geofisica e Vulcanologia, Università «Federico II», Napoli, Italy
}

\begin{abstract}
The study of Self-Potential (SP) space and time variations in volcanic areas may provide useful information on both the geometrical structure of the volcanic apparatuses and the dynamical behaviour of the feeding and uprising systems. In this paper, the results obtained on the islands of Vulcano (Eolian arc) and Ponza (Pontine archipelago) and on the Mt. Somma-Vesuvius complex are shown. On the island of Vulcano and on the Mt. Somma-Vesuvius apparatus areal SP surveys were performed with the aim of evidencing anomalies closely associated to the zones of major volcanic activity. On the island of Vulcano a profile across the fumaroles along the crater rim of the Fossa Cone was also carried out in order to have a direct relationship between fumarolic fracture migration and flow rate and SP anomaly space and time variations. The areal survey on the island of Ponza, which is considered an inactive area, is assumed as a reference test with which to compare the amplitude and pattern of the anomalies in the active areas. A tentative interpretation of the SP anomalies in volcanic areas is suggested in terms of electrokinetic phenomena, related to the movement of fluids of both volcanic and non-volcanic origin.
\end{abstract}

Key words self-potential - volcanology

\section{Introduction}

About four years ago we began a systematic study of the Self-Potential (SP) field behaviour in Italian volcanic areas, in order to obtain information on both the geometrical structure and the dynamical properties of the volcanic apparatuses.

The choice of the SP method rests on the assumption that it can fruitfully detect space and time variations of the spontaneous electric polarizations related to low and high-temperature fluid flows and, in volcanic areas, to the typical volcanic manifestations, like fumaroles, magma uprising, etc. In particular, we studied the active areas of the island of Vulcano (Eolian arc) and of the Mt. Somma-Vesuvius complex (SE of Naples), and the inactive area of the island of Ponza (Pontine archipelago).
This last experiment provided a reference test with which to compare the results in the active areas.

The SP measurements were programmed in two ways: the first approach was regional and involved areal surveys; the second one was local and involved profiles located in particularly active zones.

On the island of Vulcano we applied both techniques: the first one along circuits crossing the whole island, the second one along a circular profile, repeated many times, around the Fossa crater rim. On the island of Ponza and in the Mt. Somma-Vesuvius area we performed only areal surveys.

The regional survey aims at providing a map of the SP isolines, in order to detect anomalous areas to be connected with underground anomalous concentrations of electric charges. These concentrations are very likely provoked by underground circulation of fluids in porous media and along permeable fracture 
systems, and the physical mechanism can be explained in terms of electrokinetic phenomena. The importance of this approach is evident: in fact, knowledge of the structural configuration of the electrokinetic paths may provide indications about the buried geometries in the surveyed areas, as well as help understand the role of both water solutions and magmatic fluids and their interaction in the deep dynamics of the volcanic apparatuses.

The local profiling aims at monitoring time and space variations of the local anomalies related to the volcanic activity in progress in particularly active zones. An instructive case-history based on this last approach concerns the Mt. Etna volcano, where we performed systematic SP measurements along a profile crossing the narrow fracture system, which opened on the SE flank of the volcano in September 1989. Details of this study are reported in Di Maio and Patella (1994).

In this paper, the results of the experimental research are presented and interpreted on a qualitative basis in accord with the electrokinetic model related to fluid movement.

\section{Island of Vulcano}

\subsection{SP areal survey}

\subsubsection{Data presentation}

Figure 1 shows the survey circuitry along which we performed continuous SP measurements. We adopted a receiving dipole length of $100 \mathrm{~m}$ and used copper rod electrodes. The SP drops were detected one after the other between every two consecutive positions of the electrodes (leapfrog technique), after having reached signal stability conditions. An HP3478B digital multimeter equipped with an HP75D microcomputer was used. The adopted acquisition technique involved averaging a set of data sampled at a constant time interval over a period of a few tens of seconds.

The survey was designed in such a way as to assure at least one common point between adjacent circuits, in order to evaluate, from the SP drops across the dipoles, the point by point SP values.

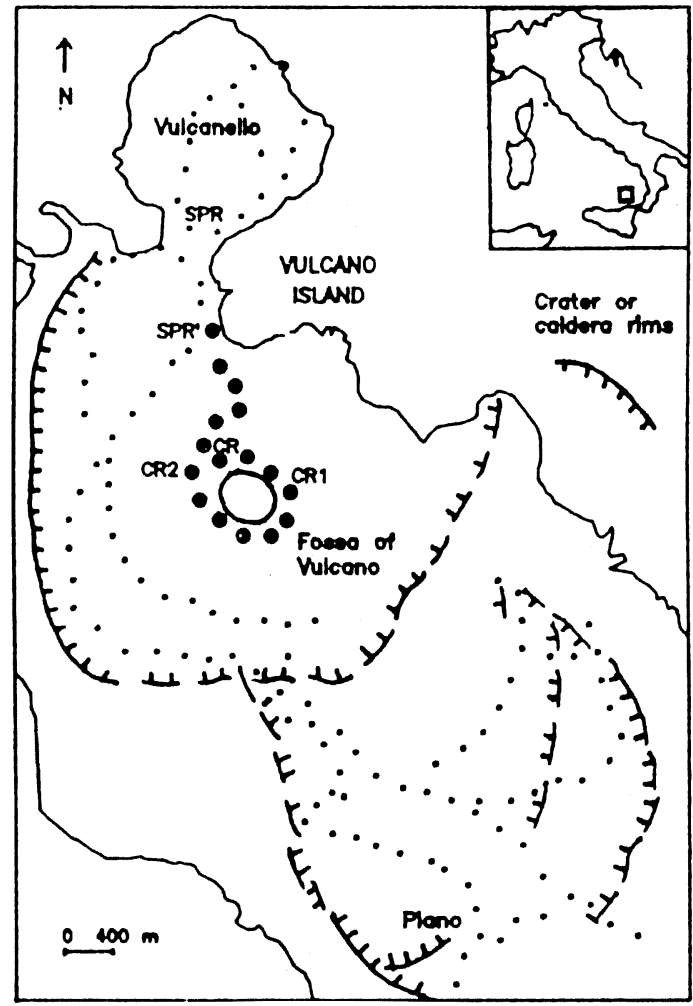

Fig. 1. Simplified tectonic sketch map, SP areal survey circuits (dots) and circular profile (circled dots) on the island of Vulcano.

In order to eliminate drift and/or locally spurious signals, the close circuits were surveyed by turning back to the initial point and the mis-tie was equally distributed over all dipolar SP drops. In the case of open circuits, the SP measurements were carried out in forward and backward directions and for each dipole the corrected SP drop was calculated by averaging the forward and backward values.

Taking into account the possible presence of electrode polarization spurious effects, we adopted the following procedure to eliminate them.

Denoting with $\alpha$ and $\beta$ the polarization voltages of the two copper rod electrodes forming the receiving dipole respectively in sites 1 and 2 along a circuit, the potential drop across the 
(a)
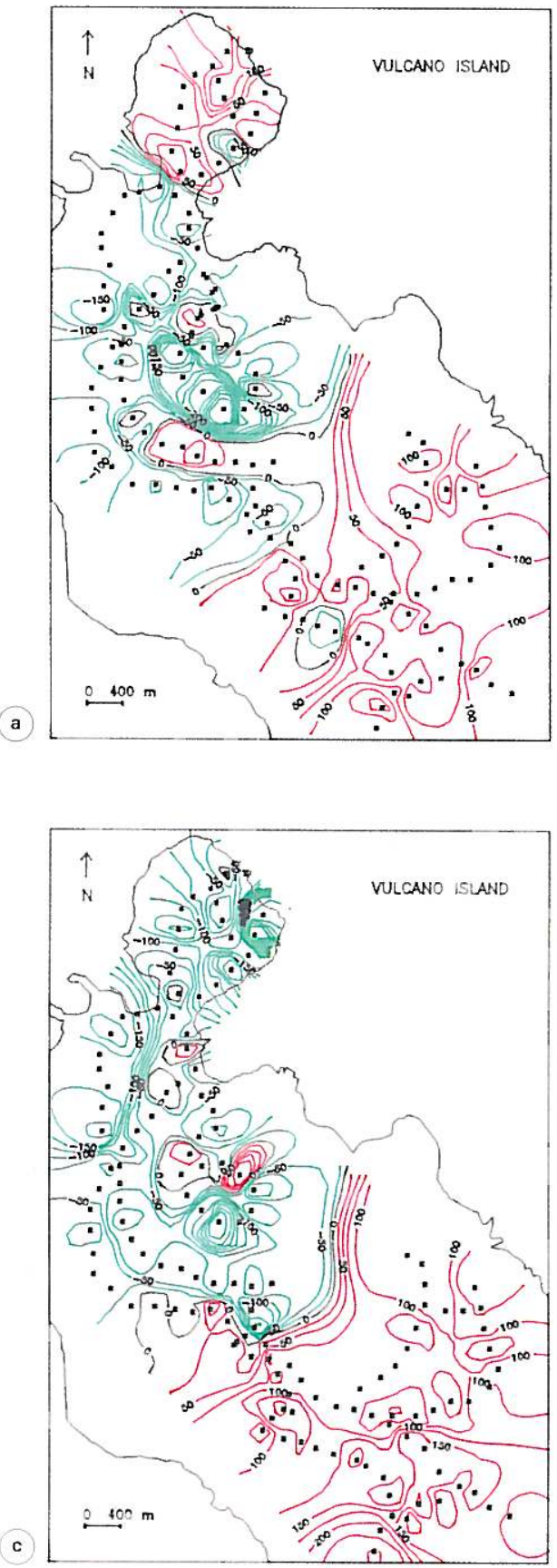

(b)
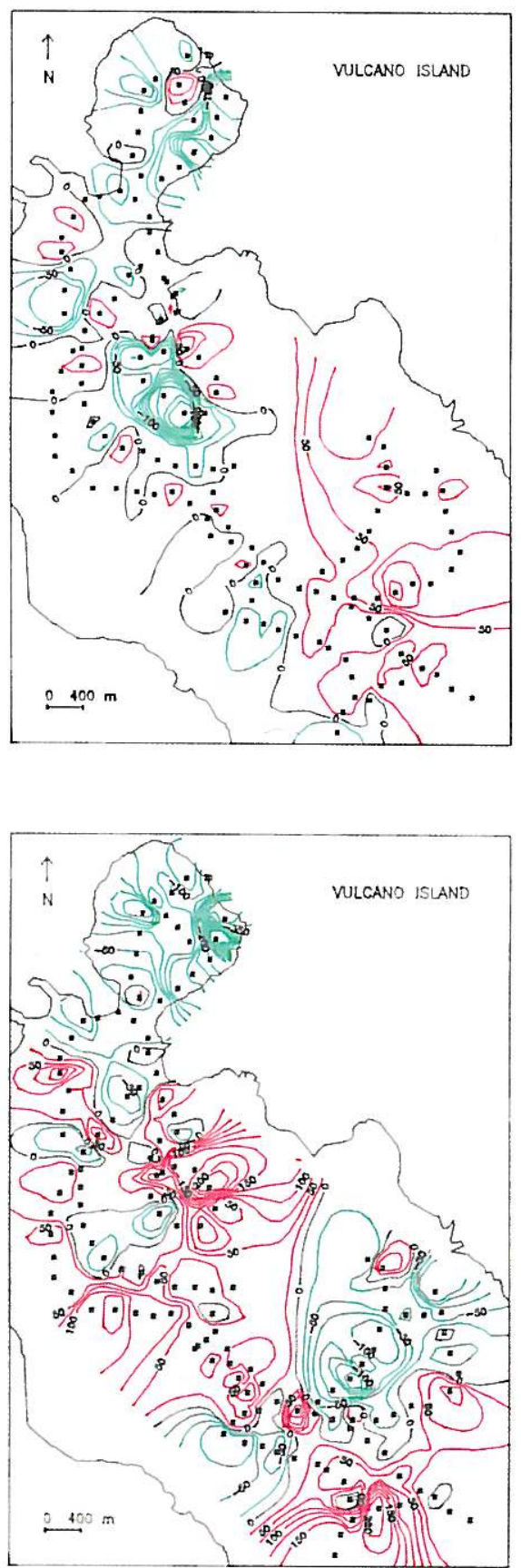

Fig. 2a-d. Maps of the SP anomalies relative to the areal surveys carried out on the island of Vulcano. Contour interval is $25 \mathrm{mV}$. Red lines are positive values, whereas green lines are negative. The black line is the zero value. a) May 1991; b) January 1992; c) June 1992; d) September 1993. 
dipole, $\delta V(2,1)$, is given by the SP drop, $\delta U(2,1)$, plus the difference between the polarization voltages of electrodes 2 and 1, viz.

$$
\delta V(2,1)=\delta U(2,1)+\beta-\alpha .
$$

If in the next contiguous dipole we alternate the role of the electrodes, by taking as fixed the electrode in site 2 and moving to site 3 the electrode previously in site 1 , we have

$$
\delta V(3,2)=\delta U(3,2)+\alpha-\beta .
$$

Supposing now that the SP potential value in site 1 is equal to zero (the arbitrary reference value), i.e. $U(1)=0$, from eq. (2.1) it readily follows

$$
U(2)=\delta V(2,1)+\alpha-\beta,
$$

which inserted in eq. (2.2), after simple steps, leads to

$$
U(3)=\delta V(3,2)+\delta V(2,1) .
$$

Iterating this procedure, one can easily deduce that all the SP values in the even positions are affected by the unknown electrode polarization difference $(\alpha-\beta)$, while all the SP values in the odd positions are electrode polarization-free. Thus, for the purposes of the analysis, only these last SP values are to be taken into consideration.

In practice, the point by point SP values in the island of Vulcano were calculated by attributing the SP zero value to the reference point denoted by SPR in fig. 1 .

For a better visualization of the anomaly contrasts which characterize the surveyed area, we preferred to generate a new SP data set, by subtracting from each value of the previously calculated set its average value.

Figure 2a-d shows the SP anomaly maps of May 1991 (a), January 1992 (b), June 1992 (c) and September 1993 (d).

\subsubsection{Data analysis and discussion}

From a qualitative point of view, the maps of fig. 2a-d show the island characterized by a low wavenumber regional trend with prevailing «negative» SP values in the northern sector and «positive»SP values in the south-eastern sector. Superimposed on this regional trend there appears a very composite pattern of high wavenumber local anomalies, the most intense of which, with sometimes reversed polarity, are those in the Vulcanello and Fossa crater areas.

As concerns the Fossa crater area, the relative variation of the intensity and width of the two nuclei forming a dipole-like local anomaly field is particularly evident. This very impressive time evolution of the dipole field surface signature can be very likely ascribed to an underground convective fluid circulation mechanism.

Another important feature, which is more or less evident in all the maps, regards the behaviour of the isolines in some transition zones from positive to negative SP values, which can be readily correlated to the main structural features of the volcanic apparatus reported in fig. 1. Indeed, one can observe very closely delineated the SP counterpart of the southern rim of the Fossa caldera. Whereas, the positive-tonegative transition in the south-eastern sector only in the map of fig. $2 \mathrm{~d}$ can be ascribed to the composite south-eastern boundary of the Piano caldera. Finally, the less evident NW-SE line marking the sign transition in the southwestern part of the map of fig. $2 b$ would delineate the western boundary again of the Piano caldera.

The geophysical model we can hypothesize to explain the general time and space pattern of the observed SP anomalies is based on electrokinetic phenomena associated with movement of underground fluids through porous media and along permeable fracture systems. We hold these phenomena responsible for anomalous concentrations of electrical charges, leading to generation of dipole-type electric fields easily detectable on the ground surface. For details on the electrokinetic polarization mechanism and its geophysical consequences, see Keller and Frischknecht (1966), Mizutani et al. (1976), Fitterman (1983) and Di Maio and Patella (1991).

Generally speaking, if the investigated areas 
are not characterized by active dynamics, the SP measured amplitudes are rather low, rarely exceeding $100 \mathrm{mV}$ according to our experience, as we shall see later in the presentation of the data collected on the island of Ponza. In active areas, instead, the SP amplitudes can be much higher. We admit that in such cases two mechanisms can explain strong electrokinetic effects. One is related to the combined action of fracturing, due to migration of a magmatic intrusion or pressurized hot fluids towards the ground surface, and invasion of the new cracks with water from nearby reservoirs, as hypothesized to justify the intense SP anomaly field across an active fracture system running along the southern slopes of Mt. Etna (Di Maio and Patella, 1994). The second mechanism is related to the forces generated by strong temperature gradients, close to hot magmatic bodies and/or fluids, which provoke water circulation in overlying porous rocks with possible formation of convective cells (Di Maio and Patella, 1991).

On the basis of this geophysical model, the analysis of the maps shown in fig. 2a-d leads to the following qualitative interpretation. The long wavelength SP pattern can be correlated to the existence of a regional polarization field, whose axis trends NW-SE and which may correspond to a horizontal sea-water flow beneath the island. The local fields, especially the one of the Fossa crater, are very likely ascribable to convective water movements, as previously outlined.

Finally, as regards the SP behaviour around the Piano caldera rims, the circumstance that the SP pattern does not always closely conform to the geometry of the caldera boundaries is not easy to understand. Different causes can be invoked in this notably less active sector of the island, among which, for instance, an increase in the rain percolation rate and/or of the seawater inland invasion flow.

\subsection{SP circular profiling}

\subsubsection{Data presentation}

The local profiling around the Fossa crater, indicated in fig. 1 by circled dots, aims at mon-

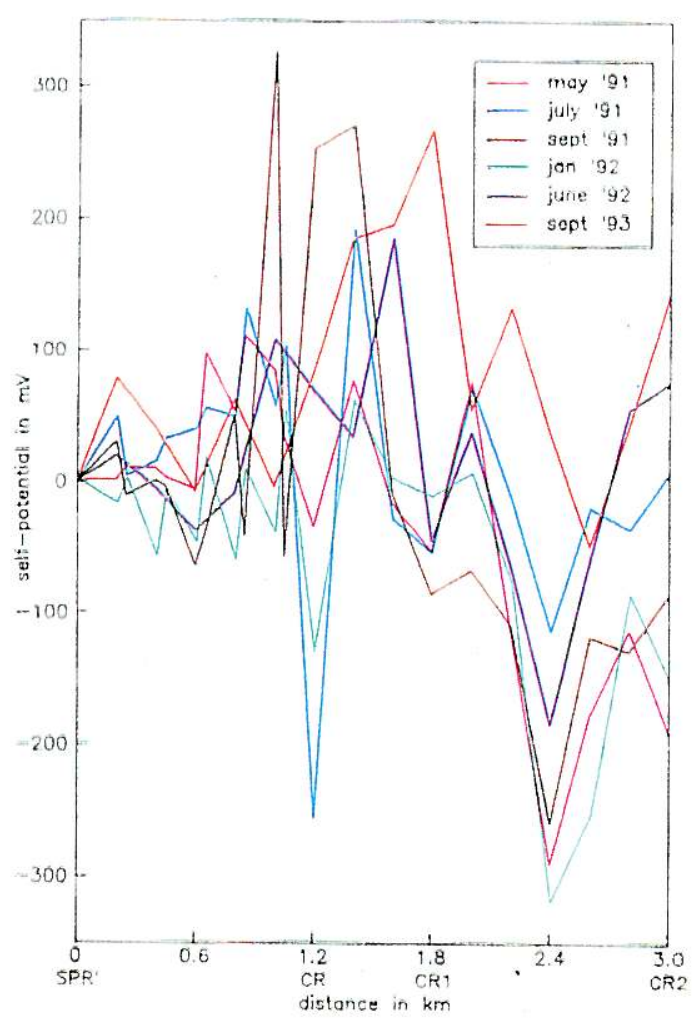

Fig. 3. Rectified SP circular profiles carried out on the island of Vulcano.

itoring time and space variations of the local anomalies related to the existing fumarolic activity, in a depth range from the surface manifestations to the shallow buried source zone.

The measuring technique and the method of data processing are the same as those used for the SP areal survey. The SP reference zero value for the repeated profiles is now the point $\mathrm{SPR}^{\prime}$ of fig. 1. In this case no further shifting of the data set was necessary.

Figure 3 shows superimposed the six profiles of May, July and September 1991, January and June 1992 and September 1993. In the figure, for the sake of easier representation, the circular profile was rectified and the sequence of points corresponds, in the first lefthand portion, to the route from site SPR' to 
site CR (see fig. 1), and, in the remaining portion, to the whole route around the crater rim covered clockwise.

\subsubsection{Data analysis and discussion}

By observing fig. 3 we point out the presence of high wavenumber anomalies superimposed on a long wavelength anomaly. This last anomaly (positive to negative from left to right), apart from different peak amplitudes, is nothing but a different visualization of the couple of anomalies shown in the maps previously discussed, which are concentrated above the same circuit. Thus, we have confirmation of this local areal feature also by the profiles of July and September 1991.

The long wavelength anomaly may be associated to a deep electrokinetic effect, which, considered the high amplitudes, could be very likely caused by strong pressure and/or temperature gradients, as previously explained in the discussion of the areal surveys.

The high wavenumber anomalies, instead, could be correlated with a superficial thermoelectric phenomenology related to hot fluid migration toward the ground surface (Fitterman, 1983). A strong temperature gradient is established between the open fractures and the surrounding walls, which produces charge displacements and, hence, the formation of an almost continuous double layer. In fact, these anomalies are essentially concentrated along the left-hand half of the rectified circular profiling, which corresponds to the sector of the Fossa crater rim affected by intense fumarolic activity with temperatures as high as $700^{\circ} \mathrm{C}$.

Finally, looking at the trend of the anomaly profiles shown in fig. 3, a further aspect deserving consideration is the migration of the high wavenumber peaks and the variation of their amplitudes. We think that the space variations of the anomalous SP peaks can be related to the sealing-opening process of the surface fractures, whereas the amplitude variation of the SP peaks may be attributed to changes in the fumarolic flow rate.

\section{Ponza}

\subsection{SP areal survey}

\subsubsection{Data presentation and analysis}

Self-potential measurements were also performed on the island of Ponza, precisely in April, 1992. As previously said, the main objective was to compare SP anomalies in active volcanic areas, such as the island of Vulcano, with those relative to inactive areas, such as the island of Ponza.

For this reason, we carried out on the island of Ponza an areal survey with the same measuring, processing and analysis techniques adopted for the areal surveys on the island of Vulcano. Figure 4 shows the survey circuitry covering the whole island. The SPR dot in fig. 4 represents the zero SP reference station.

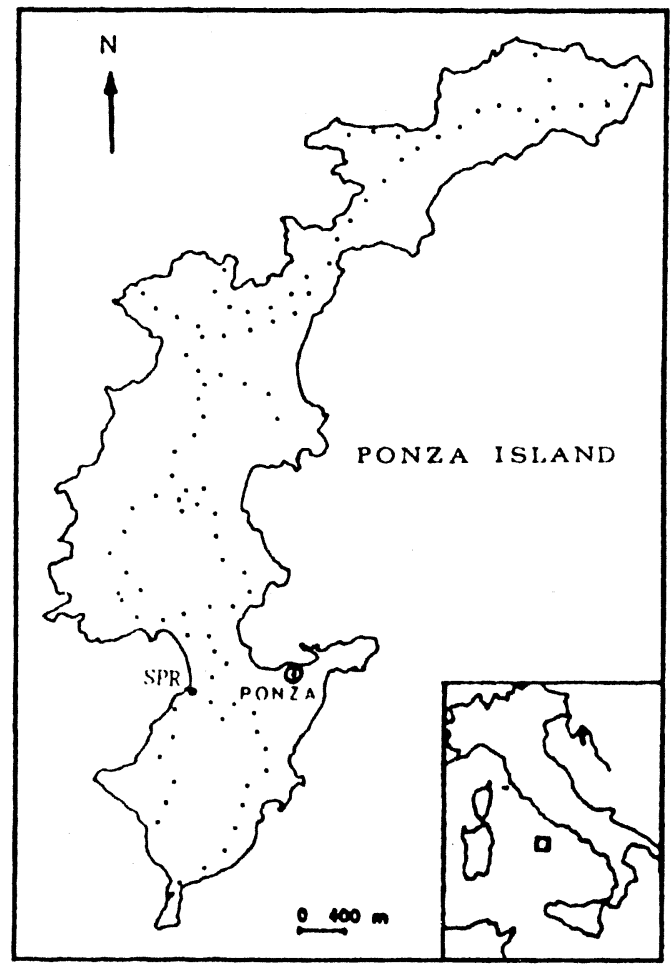

Fig. 4. SP areal survey circuits on the island of Ponza. 


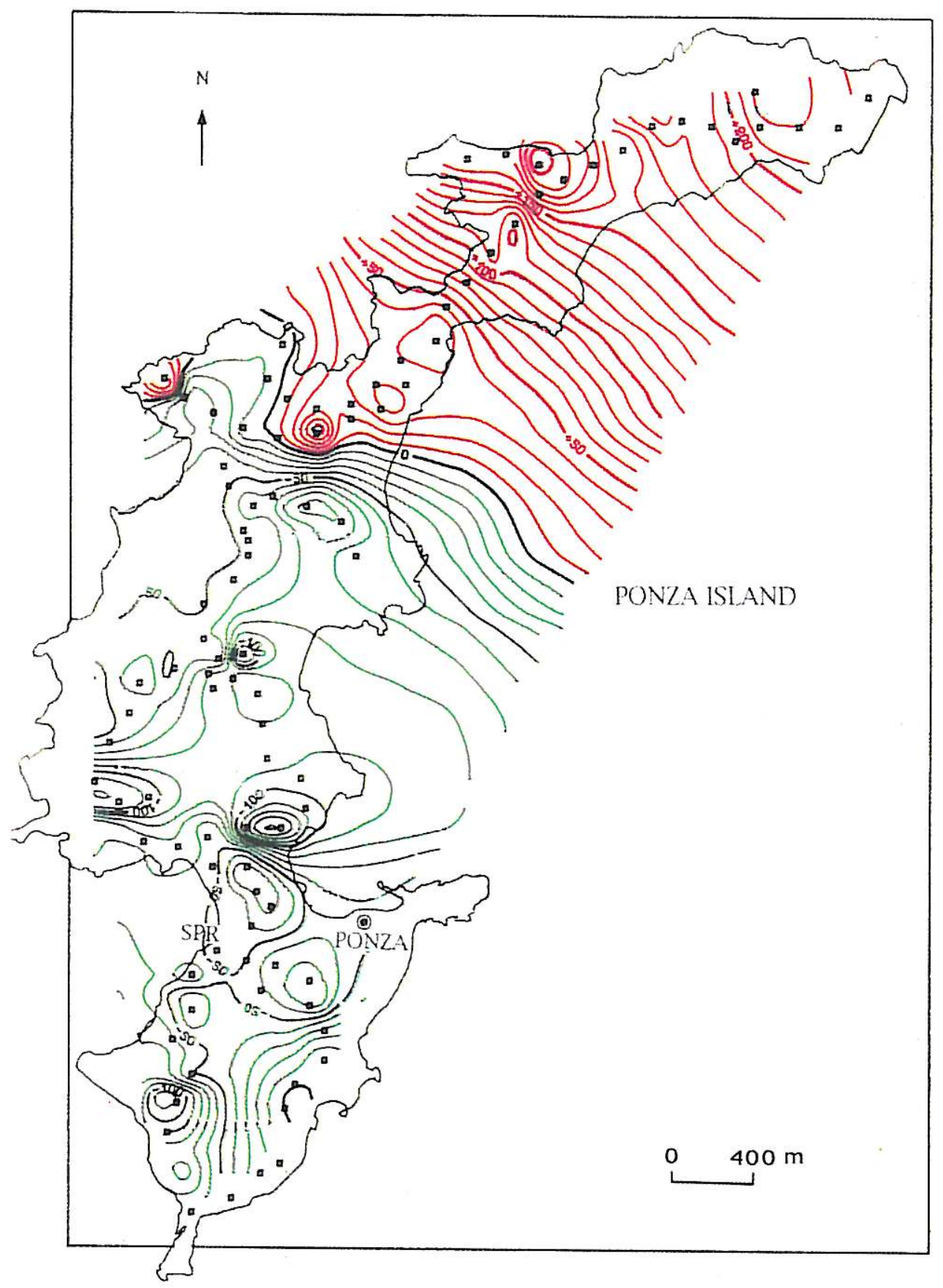

Fig. 5. Map of the SP anomalies relative to the areal survey carried out on the island of Ponza on April 1992. Contour interval is $10 \mathrm{mV}$. Red lines are positive values, whereas green lines are negative. The black line is the zero value. 
Also in this case, for a better visualization of the anomaly contrasts, the anomaly map shown in fig. 5 was drawn from the SP data set, generated by shifting the zero to the average value of the SP original data set.

Again, from a purely qualitative point of view, proceeding from north to south, a regular passage from a positive to a negative anomaly is evident, whose relative maxima seem to concentrate in correspondence with the extremities of the Island. This feature of regional character is not different from the one found on Vulcano, and it seems associated to a normal electrokinetic effect linked to the movement of sea-water beneath the island. On the contrary, as regards the highest wavenumber anomalies, the map of fig. 5 presents weak amplitudes and no significant shapes closely associated to some volcanic activity, as for the Fossa crater and Vulcanello areas at Vulcano.

\section{Mt. Somma-Vesuvius}

\subsection{SP areal survey}

\subsubsection{Data presentation}

In the volcanic area of Mt. Somma-Vesuvius we carried out SP measurements according to the areal survey approach.

The inaccessibility of many zones on the upper flanks of the volcano and the heavy urbanization downhill prevented us using the measuring technique adopted for the regional surveys on the islands of Vulcano and Ponza. Therefore, we chose an alternative technique consisting in the determination of the electric field components in the EW and NS directions, in a set of stations spaced on the average about $500 \mathrm{~m}$ (fig. 6). This prospecting, considering the vastness of the area (about $150 \mathrm{~km}^{2}$ ) as well as the remarkable morphological and logistic difficulties, took about two months of field work, precisely from June to July 1992. We adopted a receiving dipole length of $10 \mathrm{~m}$ and used grounded copper-copper sulphate impolarizable electrodes.

Figures 7 and 8 show the anomaly maps of the $E_{x}(\mathrm{EW})$ and $E_{y}(\mathrm{NS})$ components, respectively.

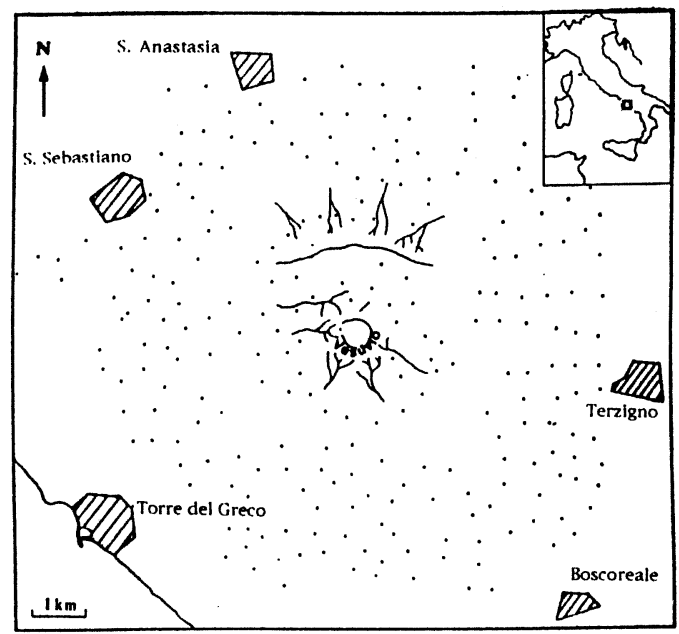

Fig. 6. Station sites (dots) for the measurements of the natural electric field EW and NS components in the Mt. Somma-Vesuvius area.

\subsubsection{Data analysis and discussion}

Looking at the maps of figs. 7 and 8, we observe in the southern sector of the volcano the presence of a large number of high wavenumber positive and negative anomalies, whose amplitudes sometimes reach very high values (about $20-25 \mathrm{mV} / \mathrm{m}$ ). It is also important to note the wide high-amplitude anomaly distribution in correspondence with the Vesuvius cone summit.

Even though the anomaly trend of the natural electric field components is rather articulated and complex, we give a tentative qualitative interpretation on the basis of the previously proposed geophysical model.

The asymmetric distribution of the anomalies between the northern and southern sectors of the investigated area could be attributed to the gradual vanishing of volcanic activity in the area of Mt. Somma (northern part) and to the persisting activity around the Vesuvius volcanic apparatus. In fact, the Vesuvius sector, contrary to the Mt. Somma zone, is still subject to deep gaseous flows (essentially $\mathrm{CO}_{2}$ ) and thermal anomalies (Bellucci et al., 1993). 


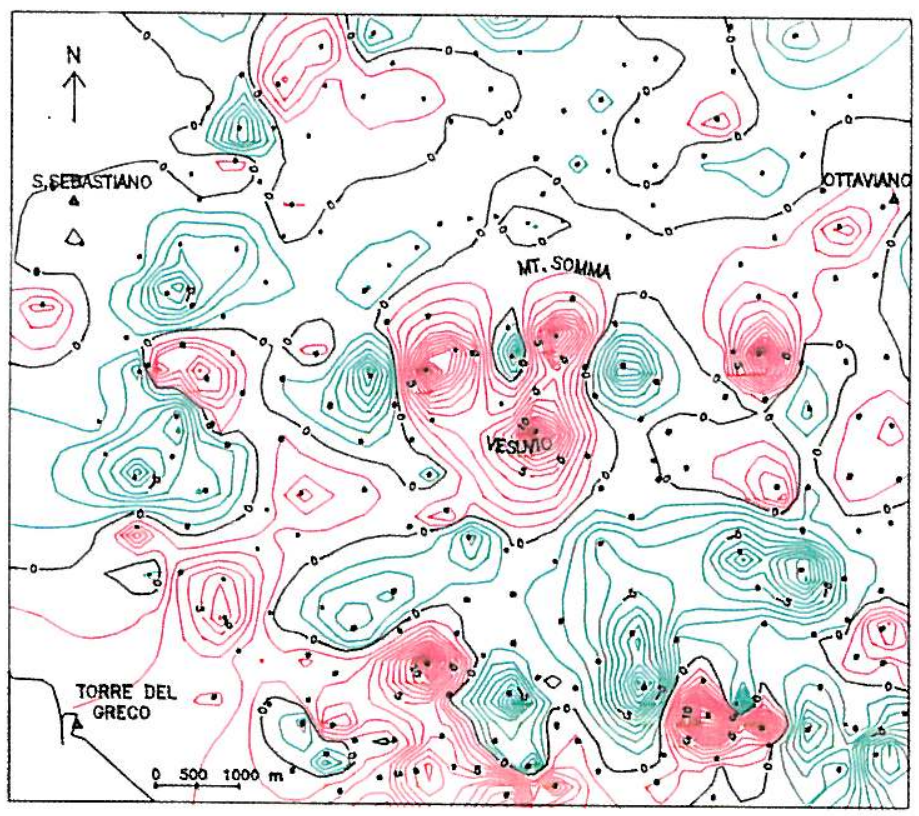

Fig. 7. Map of the anomalies of the EW component of the natural electric field $\left(E_{x}\right)$ in the Mt. SommaVesuvius area. Contour interval is $1 \mathrm{mV} / \mathrm{m}$. With the horizontal axis directed east-westwards, red lines are positive values, whereas green lines are negative. The black line is the zero value.

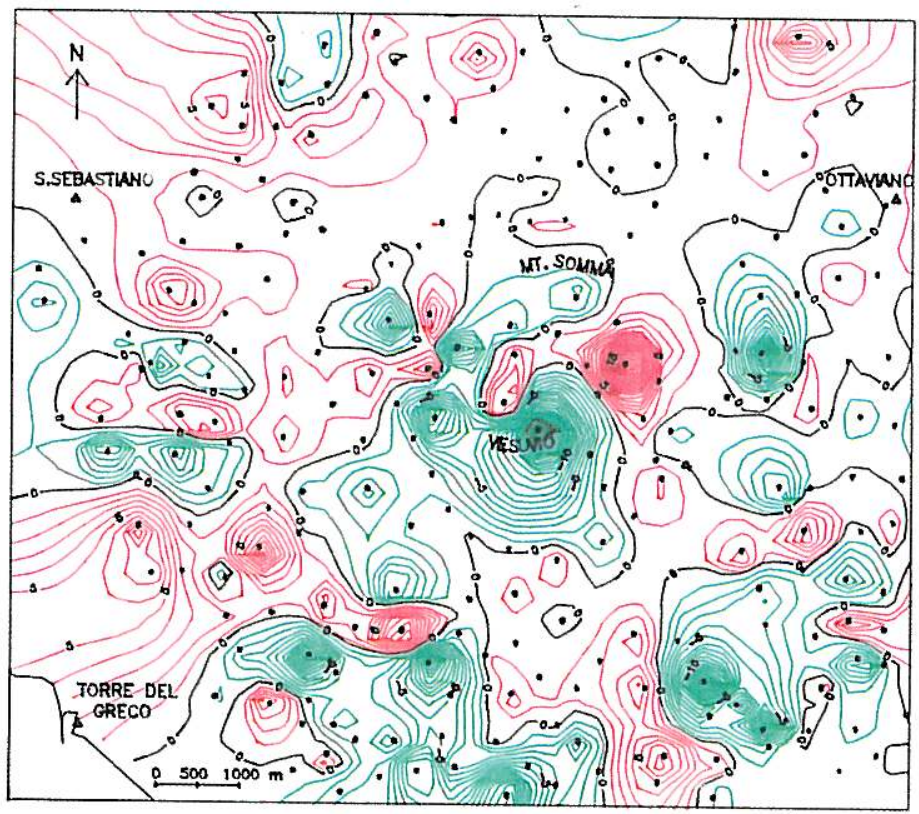

Fig. 8. Map of the anomalies of the NS component of the natural electric lield $\left(E_{y}\right)$ in the Mt. SommaVesuvius area. Contour interval is $1 \mathrm{mV} / \mathrm{m}$. With the vertical axis directed north-southwards, red lines are positive values whereas green lines are negative. The black line is the zero value. 
As concerns the high wavenumber negative and positive anomalies around the axis Boscoreale-Camaldoli-S. Sebastiano (see figs. 7 and 8), we can hypothesize the presence of preferential paths of fluid outflows. In fact, the transition zones from positive to negative anomalies could indicate the probable existence of fractures. This hypothesis seems to conform to the results of hydrogeological and hydrogeochemical researches (Celico et al., 1994; Bellucci et al., 1993) carried out in the southern sector of the Somma-Vesuvius apparatus. These studies have delineated the water circulation scheme underground. The relative high salinity of the waters, the high content in $\mathrm{HCO}_{3}$, the areal distribution of $\mathrm{CO}_{2}$, the presence in water of tracings from deep circuits and of good indicators of thermal anomalies, like $\mathrm{Li}$ and $\mathrm{B}$, have been interpreted, in fact, as the result of fluid uprising from the calcareous basement. The preferential uprising paths seem to be concentrated just in the area where we observed the large high wavenumber anomaly distribution, that is in the southern sector of the volcano.

The presence of the high amplitude negative and positive anomalies in the central part of the maps of figs. 7 and 8, that is in the Vesuvius summit area, could instead be attributed to the local fumarolic activity, and also to the existence of fractures and deep vents affected by electrokinetic phenomena related to fluid dynamics, as was previously outlined in the discussion of the Vulcano island results.

Finally, the lack of pronounced anomalies in the northern sector of the volcanic apparatus seems to indicate the lack of fluid uprising. This is also confirmed by the above mentioned hydrogeological and hydrogeochemical studies. Therefore, the weak variations of the natural electric field, observed in this sector, may be associated to the downflow of the underground waters.

\section{Conclusions}

Self-potential anomalies observed in three Italian volcanic areas (islands of Vulcano and Ponza and Mt. Somma-Vesuvius complex) have been presented and discussed.
The proposed geophysical models, essentially based on the electrokinetic mechanisms related to fluid movements, have permitted the SP space variations to be correlated with the geometrical characteristics of the investigated areas. The SP time variations, observed by repetition of the regional survey and by local profiles, besides giving some new information for a better delineation of the structural setting of the volcanic complexes, seem to be well correlated with the evolution of the dynamic processes, the most important of which for the generation of SP anomalies are the presence of sharp pressure and temperature gradients, underground water and gas circulation rate, fumarolic outflows, magma bodies and fluids uprising and tectonic activity.

\section{Acknowledgements}

Study performed with financial aid from the Italian Volcanological Group, National Research Council and Civil Defense Department, Rome, Italy.

\section{REFERENCES}

Bellucci, F., A. Corniello and R. De Riso (1993): Geology and hydrogeology of Somma-Vesuvio Volcano (Southern Italy), I.A.H. Memories, 24 (1), 137-149.

Celico, N., P. Celico, M.R. Ghiara, V. Piscopo, D. STANZIONE and S. AQUiNo (1994): Caratteristiche geochimiche delle acque sotterranee dell'area del Somma-Vesuvio (Campania, Italia), Atti II Conv. Naz. Ric. Geol. Appl., Viterbo, Geologica Romana, 30, 709-724.

Di MAio, R. and D. PATElla (1991): Basic theory of electrokinetic effects associated with earthquakes, Boll. Geof. Teor. Appl., 33, No. 130-131, 145-154.

Di MAio, R. and D. PATElla (1994): Self-potential anomaly generation in volcanic areas. The Mt. Etna case-history, Acta Vulcanologica, 4, 119-124.

FITTERMAN, D.V. (1983): Modeling of self-potential anomalies near vertical dykes, Geophysics, 48, 171-180.

KELLER, G. V. and F.C. FRISCHKNECHT (1966): Electrical Methods in Geophysical Prospecting (Pergamon, New York).

Mizutani, H., T. IshIDO, T. YoKOKURA and S. OHNISHI (1976): Electrokinetic phenomena associated with earthquakes, Geophys. Res. Lett., 3, 365-368. 